\title{
De Villoldo à Gardel
}

Changement de rythme et effets poétiques dans la chanson tango

From Villoldo to Gardel: Change of Rhythm and Poetic Effects in the Tango Song

\section{Pénélope Patrix}

\section{(2) OpenEdition}

\section{Journals}

Édition électronique

URL : https://journals.openedition.org/clo/2036

DOI : $10.4000 /$ clo.2036

ISSN : 2266-1816

Éditeur

INALCO

Édition imprimée

Date de publication : 1 janvier 2013

ISBN : 978-2-85831-217-7

ISSN : 0396-891X

Référence électronique

Pénélope Patrix, «De Villoldo à Gardel », Cahiers de littérature orale [En ligne], 73-74 | 2013, mis en ligne le 11 mai 2015, consulté le 01 juillet 2021. URL : http://journals.openedition.org/clo/2036 ; DOI : https://doi.org/10.4000/clo.2036

Ce document a été généré automatiquement le 1 juillet 2021

\section{(c) (†) \&)}

Cahiers de littérature orale est mis à disposition selon les termes de la Licence Creative Commons Attribution - Pas d'Utilisation Commerciale 4.0 International. 


\title{
De Villoldo à Gardel
}

\author{
Changement de rythme et effets poétiques dans la chanson tango \\ From Villoldo to Gardel: Change of Rhythm and Poetic Effects in the Tango Song
}

\section{Pénélope Patrix}

1 Les premières interprétations par Carlos Gardel en 1917 de tangos tristes et lents, aux paroles amères, constituent une innovation majeure dans l'histoire du tango chanté. Avant lui, Ángel Villoldo et Alfredo Gobbi, les chanteurs caractéristiques du tango, ont cultivé un style vif et enjoué inspiré du théâtre populaire. Gardel impose son tango mélancolique en modèle normatif: il invente et institue la manière de chanter, dorénavant, le tango. Celui-ci est alors défini par la critique comme une chanson triste par essence, et les chansons gaies sont reléguées à la "préhistoire " du tango chanté. Pour comprendre cette innovation, il est intéressant de comparer les enregistrements de Villoldo et de Gardel. Nous verrons qu'au cœur de cette transformation se trouve un basculement d'un type de rythme à un autre. En effet, outre des modifications localisées, c'est l'agencement même du «rythme » entendu au sens large qui est bouleversé. Le chanteur impose un flux qui lui est propre, singulier et imprévisible, et la régularité rythmique, qui prévalait, est déconstruite. Ce changement de rythme est corrélé à des transformations poétique, musicale, vocale et performative importantes. Simultanément, le statut de la chanson tango évolue: à partir de Gardel elle est intégrée au répertoire de cabarets élégants des centres-villes de Buenos Aires et de Montevideo et instituée en chanson raffinée ${ }^{1}$. La vente de disques augmente rapidement avec l'engouement du grand public pour le tango gardélien, tandis que le tango " allègre » décline. Si la critique considère unanimement la création de Mi Noche Triste comme un point de rupture - moment où le tango-chanson serait inventé, autrement dit comme l'invention d'un genre nouveau ${ }^{2}$ - nous proposons quant à nous de parler d'innovation majeure au sein d'une tradition déjà vivante. En effet, l'analyse permettra d'identifier de Villoldo à Gardel des éléments communs qui inscrivent ce dernier dans la continuité de ses prédécesseurs, et permettent d'envisager le changement de rythme plutôt comme un « passage $»^{3}$.

2 Cette innovation poético-rythmique sera abordée à travers les interprétations enregistrées de deux chansons emblématiques, El Pechador et Mi Noche Triste. Nous 
évaluerons ces changements rythmiques et stylistiques à l'aune du «rythme» de la performance dans son ensemble et de la configuration de la relation entre chanteur et auditeurs.

\section{Le tango allègre d'Ángel Villoldo : El Pechador}

3 Ángel Villoldo (1861-1919) a chanté en s'accompagnant à la guitare et à l'harmonica dans divers lieux festifs de Buenos Aires : fêtes des faubourgs, cafés, guinguettes et cafés-concerts. Il est le premier à écrire dès 1890 des chansons appelées «tango » qui soient pensées comme un tout parole-musique et séparées des pratiques instrumentales. De plus, ses chansons sont «territorialisées » dans la culture portègne ${ }^{4}$ par leurs sujets locaux, leurs parlures, leurs syncrétismes rythmiques propres à la musique criolla, ce qui les distingue des tangos à l'espagnole en vogue à Buenos Aires à l'époque. Nous pouvons les considérer, en fait, comme les premières chansons de tango criollo. Son vaste répertoire est facilement identifiable, car les mélodies et rythmes sont quasiment identiques d'une chanson à l'autre, et ses paroles réalistes toujours écrites selon le même principe, où un voyou des faubourgs vante ses exploits canailles dans un registre "allègre » 5 . La chanson El Pechador («Le Tapeur ») est écrite vers 1905. Nous travaillons ici sur le second enregistrement datant de 1909-19106 , où Villoldo est accompagné par un orchestre de type fanfare (avec tuba, trombone et/ou trompette, clarinette, deux flûtes dont une soprano, et des percussions). Malgré les déformations importantes de l'enregistrement ${ }^{7}$, nous avons transcrit les paroles suivantes, les « trous » étant indiqués entre crochets.

\begin{tabular}{|c|c|c|c|}
\hline $\begin{array}{l}\text { Section } \\
\text { music. }\end{array}$ & $\begin{array}{l}\text { Couplet et strophe } \\
\text { (X.x) }\end{array}$ & Paroles & Traduction \\
\hline 0 & & $\begin{array}{l}\text { (introduction } \\
\text { instrumentale) }\end{array}$ & \\
\hline 1 & A.a & $\begin{array}{l}\text { Yo me llamo Fortunato } \\
\text { mi apellido es Peñaflor } \\
\text { conocido en todo el barrio } \\
\text { por el primer pechador, } \\
\text { de los pesaos soy el taita } \\
\text { y al que quiera balancear, } \\
\text { pelando mi fariñera ichulinga! } \\
\text { lo hago al momento espiantar. }\end{array}$ & $\begin{array}{l}\text { Je m'appelle Fortunato } \\
\text { mon surnom est Peñaflor } \\
\text { connu dans tout le quartier } \\
\text { comme le plus grand des tapeurs, } \\
\text { des casse-pieds je suis le caïd } \\
\text { et celui qui veut me défier, } \\
\text { en dégainant mon couteau - flûte! } \\
\text { je le fais fuir sur le champ. }\end{array}$ \\
\hline 2 & B.a & $\begin{array}{l}\text { Como resorte, soy pa'l cuchillo } \\
\text { y no hay chimango que me las } \\
\text { dé, } \\
\text { y el que ha querido meterse a } \\
\text { guapo } \\
\text { con dos planazos lo abataté. } \\
\text { [4 vers ] }\end{array}$ & $\begin{array}{l}\text { Pour ressource, j'ai mon couteau } \\
\text { et y'a pas d'écervelé qui m'la fait, } \\
\text { et celui qui a voulu faire le malin, } \\
\text { de deux coups je l'ai abattu. } \\
\text { [...] }\end{array}$ \\
\hline
\end{tabular}




\begin{tabular}{|c|c|c|c|}
\hline 3 & $\begin{array}{l}\text { C.a } \\
\text { C.b }\end{array}$ & $\begin{array}{l}\text { En este mundo, señores, } \\
\text { yo lo puedo asegurar, } \\
\text { muchos viven del pechazo } \\
\text { y algunos de calotear. } \\
\text { Yo no soy manco y me atengo } \\
\text { a lo que dice el refrán: } \\
\text { el vivo vive del zonzo } \\
\text { y el zonzo de trabajar! }\end{array}$ & $\begin{array}{l}\text { En ce monde, messieurs, } \\
\text { je peux vous l'assurer, } \\
\text { beaucoup vivent de l'escroquerie } \\
\text { et quelques-uns de la fraude } \\
\text { Moi je n'suis pas un pigeon et } \\
\text { j'm'en tiens } \\
\text { à ce que dit le refrain: } \\
\text { le malin vit de l'idiot } \\
\text { et l'idiot de son travail! }\end{array}$ \\
\hline 0 & & (interlude) & \\
\hline $1^{\prime}$ & D & [1 couplet] & {$[\ldots]$} \\
\hline $2^{\prime}$ & E & [ 1 couplet] & {$[\ldots]$} \\
\hline 3 & C'.a* & $\begin{array}{l}\text { Hoy [...] } \\
\text { lo que quiero es la verdad: } \\
\text { unos viven del pechazo } \\
\text { y otros de trabajar. }\end{array}$ & $\begin{array}{l}\text { Aujourd'hui [...] } \\
\text { je n'veux qu'la vérité : } \\
\text { certains vivent de l'escroquerie } \\
\text { et d'autres de leur travail. }\end{array}$ \\
\hline & $C^{\prime} \cdot b^{\prime}$ & $\begin{array}{l}\text { Yo no soy manco y me atengo } \\
\text { A lo que dice el refrán: } \\
\text { El vivo vive del zonzo } \\
\text { y el zonzo de trabajar! }\end{array}$ & $\begin{array}{l}\text { Moi je n'suis pas un pigeon et } \\
\text { j'm'en tiens } \\
\text { à ce que dit le refrain: } \\
\text { le malin vit de l'idiot } \\
\text { et l'idiot de son travail! }\end{array}$ \\
\hline
\end{tabular}

\section{Une chanson cyclique, fondée sur la répétition}

4 La mélodie, composée sur un mode majeur, est formée de thèmes populaires cousus et arrangés par Villoldo (Novati, 2008). Trois sections musicales, de durée égale, se suivent et l'ensemble est répété à l'identique (seules les paroles changent, voir infra). S'en dégage un rythme macrostructurel cyclique qui pourrait être repris indéfiniment.

5 L'accompagnement par l'orchestre apporte un soutien principalement mélodique : la flûte et la clarinette redoublent la mélodie du chant intégralement, et les cuivres à la fondamentale et à l'octave, ce qui produit un effet de redondance entre la partie chantée et la partie instrumentale ${ }^{8}$. On remarque en outre un certain ralentissement de la cadence au moment de l'entrée de la voix, confirmant que c'est elle qui régit la chanson. Nous voyons là aussi un indice de l'autonomisation de cette dernière vis-à-vis de la musique instrumentale et de la danse. La matrice rythmique correspond à la cellule habanera, faiblement marquée par la basse (trombone et percussions), mais perceptible ${ }^{9}$, avec une syncope typique du tango portègne jouée à intervalles réguliers. Cette musique répétitive et binaire est propre à encourager des pas de danse ou, du moins, une participation active de l'auditeur par la mémorisation immédiate en situation de fête ou de bal. 


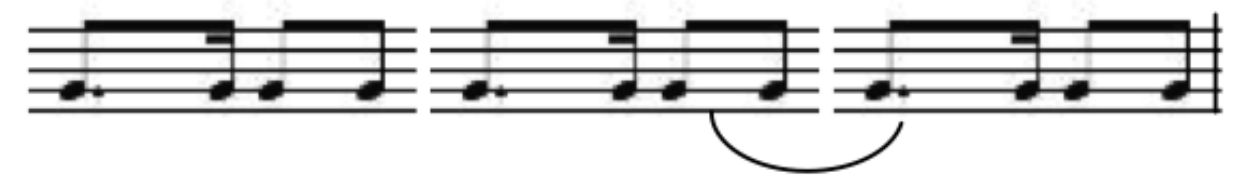

Cellule rythmique de la habanera avec syncope tanguera

6 Le poème chanté est lui aussi de forme cyclique et régulière. Il est composé d'octosyllabes, type de vers commun dans la poésie populaire du rio de la Plata. Six couplets constitués de huitains se suivent. Ce sont des quatrains associés par deux (d'où notre subdivision de chaque couplet en deux). On observe que le couplet $C$ est une répétition de $\mathrm{C}$ : d'abord simple reformulation des mêmes énoncés $\left(C^{\prime} \cdot \mathrm{a}^{*}\right)$, puis répétition exacte $\left(c^{\prime} \cdot b^{\prime}\right)$. Ce couplet peut donc être identifié comme le refrain, retour du même qui renforce l'effet de répétition et de récurrence. Ce refrain s'énonce d'ailleurs explicitement comme tel (lo que dice el refrán, voir C.b), par un procédé qu'on peut appeler métavoca ${ }^{10}$. Dicton, règle de vie, il formule une assertion ayant valeur de vérité générale et répétable à loisir.

\section{Registre « allègre » et théâtralisation des faubourgs}

7 Le texte de la chanson est comique, il vise avant tout à faire rire. De nombreux mots familiers, très imagés, créent des effets de pittoresque, mais l'effet comique provient de l'interprétation poético-musicale dans son ensemble : le registre « allègre » s'appuie sur le comique de mots, mais aussi sur divers jeux sonores. On note par exemple une isotopie de l'oiseau, logée à différents niveaux de la chanson: lexical, avec chulinga, terme lunfardo qui est littéralement un nom d'oiseau (oiseau moqueur), employé ici comme interjection dénotant l'agacement. Le mot chimango, trois vers plus bas, est aussi un nom d'oiseau (de proie) qui, en lunfardo, désigne de façon péjorative une personne désagréable ${ }^{11}$. Sur le plan musical, la flûte soprane domine l'accompagnement. Dans trois vers du premier couplet, les deux dernières syllabes nettement détachées sont immédiatement suivies de deux notes projetées à la flûte au même rythme, faisant écho à la voix de façon mimétique. Ce son aigu, qui peut évoquer un sifflement d'oiseau, agit comme un commentaire ironique ou, du moins, cocasse des paroles chantées. Ce redoublement de la voix par l'instrument produit une dramatisation du texte: une image verbale et acoustique du "Tapeur» est fabriquée, portrait burlesque d'un personnage plein d'entrain dans les rues de son quartier. C'est dans la même veine que s'inscrit le refrain provocateur. Le Pechador y prône une philosophie de profiteur. La portée burlesque de ce défi à la morale commune est manifestée par un chiasme sémantique (El vivo [a]vive del zonzo [b]/y el zonzo [b] detrabajar [a]) et une double allitération en [v] et [z], qui mettent en relief la dextérité langagière de ce beau-parleur vivotant au détriment des naïfs. Par ce retournement ironique des valeurs communes, le travailleur devient le bouffon et Fortunato s'impose en personnage comique.

8 C'est le portrait d'un voyou-type des faubourgs qui est donné, comme le souligne le titre de la chanson par l'article défini el généralisant. Ce personnage stéréotypé est présenté à la première personne du singulier, mais il est mis à distance par la caricature et l'ironie. La somme des chansons de Villoldo constitue ainsi un répertoire 
de caractères où chaque personnage s'inscrit dans le cadre d'une série. Pas de profondeur du personnage, c'est du côté de la satire de mœurs de la comédie légère comme la zarzuela ${ }^{12}$ que l'on trouverait un modèle de représentation. Tous les éléments $\mathrm{du}$ système poétique «méta-canaille $~^{13}$ de Villoldo sont rassemblés. Un univers faubourien est mis en mots et en musique, tout en étant coupé de toute référencialité. Le « je »-personnage vante ses exploits de façon provocatrice et revendique un code de mauvaise vie (actions illicites à l'instar du vol, code de l'honneur poussant à dégainer son couteau en cas de provocation); mais la théâtralité et l'écart ironique inséré dans l'énonciation permettent de distinguer le chanteur réel (Villoldo) du «je »-chanté. De plus, l'argot, s'il sert un effet de réel, est aussi manipulé de façon poétique, permettant des jeux polysémiques à visée comique, comme nous l'avons vu. La chanson est ainsi reconnue par l'auditeur comme une fiction où le chanteur se «met en scène ». Cette théâtralisation de la marginalité faubourienne est toujours effectuée dans une tonalité joyeuse, allègre, parfois grivoise.

\section{Homologie entre chant et musique, régularité du phrasé}

9 En somme, une euphonie se dégage de cette chanson gaie, redondante et régulière. L'interprétation de Villoldo renforce cet effet par une totale homologie rythmique entre musique, parole et chant.

10 Tout d'abord, son phrasé est syllabique (une syllabe par note), un syllabisme presque exagéré par sa prononciation très articulée des mots comme martelés sur la musique. De plus, la partie chantée est encore une variante de la formule rythmique habanera. Enfin, on trouve une stricte isochronie entre la métrique, la musique et le phrasé : chaque vers chanté coïncide avec une phrase musicale et chaque couplet avec une section musicale.

11 La déclamation du vers de Villoldo peut être considérée comme une forme de parléchanté : il mélodise la phrase et sa prosodie est rythmée en musique, mais il récite dans un «style scandé $»^{14}$ qui souligne les vers, tout en accentuant les mots d'une façon qui se rapproche de la langue parlée. La première syllabe du vers démarre toujours à contretemps de la mesure (et non sur le premier temps fort). Ce décalage régulier toujours placé au même endroit - respecte le placement de l'accent tonique dans la langue parlée. Mais quand celui-ci entre en concurrence avec la scansion syllabique, Villoldo privilégie cette dernière. Par exemple au mot pelando (vers 7), l'accent tonique est déplacé de la pénultième à la dernière syllabe. Il utilise en outre des procédés empruntés à la communication orale, comme l'interjection ¡Chulinga! ou la prononciation parlée de taita (1.5). L'accompagnement est symétrique à cette parole mélodisée, puisque les instruments de musique semblent "parler » : quand ce n'est pas la flûte qui répond au chanteur (voir supra), c'est la clarinette qui, du point de vue mélodique, conclut la période à sa place en l'amenant à sa résolution (vers 4 de la section 1). L'interprétation de Villoldo présente une véritable mise en scène de la voix, qui va du style parlato aux « réflexions » de l'orchestre, et où l'on reconnaît encore la dramaturgie vocale de la zarzuela. Villoldo, quand il chante le personnage du Pechador à la première personne du singulier, joue un rôle ${ }^{15}$.

12 Finalement, la chanson El Pechador peut être placée sous le signe de l'entrain. Cadence rapide, musique cyclique et répétitive, retour du refrain, phrasé régulier et uniforme permettent une mémorisation immédiate, une reprise, un "entraînement» de 
l'auditoire. Tous ces ressorts semblent en effet permettre à Villoldo d'obtenir l'adhésion de son public et l'inviter à le suivre. La relation au public est organisée avant tout autour du texte, dont l'enjeu est de faire rire l'assemblée. La chanson est une adresse directe au public, à caractère fortement dialogique, le «je " mis en scène s'adressant directement aux señores (C.a) qui l'écoutent. Nous pouvons imaginer une assemblée effectivement majoritairement masculine à l'époque, riant ou souriant dans la salle d'un des cafés où chante Villoldo, dans la rue d'un quartier populaire, ou encore dans une salle des fêtes munie d'un gramophone où elle écoute ce disque entre deux danses. La régularité formelle observée rejoint l'enjeu performatif, articulé autour de la fête.

\section{Carlos Gardel et la fêlure du rythme: Mi Noche Triste}

Mi Noche Triste est quant à elle une création à trois têtes : le poète Pascual Contursi écrit des paroles sur le tango Lita composé en 1915 par le musicien Samuel Castriota pour son trio, mais c'est Carlos Gardel qui rend la chanson célèbre. Il se produit à cette époque dans un théâtre de Buenos Aires, fréquenté par la bourgeoisie de la ville, où il chante des chansons sentimentales folkloriques. Il décide d'inclure ce tango dans son répertoire, choix inouï (et risqué), car le genre est considéré comme vulgaire par les élites; mais il remporte un franc succès (Novati, 2008). Nous travaillons ici sur l'enregistrement réalisé en 1917 avec José Ricardo à la guitare ${ }^{16}$. Dès lors une dissociation a lieu entre le tango « canaille » des fêtes populaires (et ses adaptations au théâtre) et le tango mélancolique et méditatif des lieux élégants, qui s'écoute assis.

C'est la première différenciation "rythmique » opérée au niveau de la performance tanguera dans son ensemble : chanté pour un public bourgeois, dans des configurations de séparation scène/salle, le rythme de la chanson est libéré des contraintes de l'entrain. Au « rythme debout » de la fête se substitue le «rythme assis » du cabaret. La chanson n'est plus intégrée à un évènement festif, comme l'était le tango allègre, et la performance ne vise pas à être collectivement incorporée (embodied) ${ }^{17}$. Ce "rythme assis » correspond à une position du public, moins participant (ou co-énonciateur) que spectateur. Gardel offre le spectacle inimitable, virtuose et unique de son interprétation qui plus est d'une chanson lyrique, intime. La relation au public ne se construit pas par l'adhésion, mais par une identification possible.

\begin{tabular}{|l|l|l|l|}
\hline Sectionmusic & Couplet & Paroles & Traduction \\
\hline 1 & & (intro. instrumentale) & \\
\hline 1 & A & $\begin{array}{l}\text { Percanta que me amuraste } \\
\text { en lo mejor de mi vida } \\
\text { dejándome el alma herida } \\
\text { yespina en el corazón, } \\
\text { sabiendo que te quería } \\
\text { que vos eras mi alegría } \\
\text { ymi sueño abrasador } \\
\text { para mí ya no hay consuelo } \\
\text { ypor eso me encurdelo } \\
\text { pa' olvidarme de tu amor. }\end{array}$ & $\begin{array}{l}\text { Fille tu m'as abandonné } \\
\text { au meilleur moment de ma vie } \\
\text { me laissant l'âme blessée } \\
\text { et une épine dans le cœur } \\
\text { sachant que je t'aimais } \\
\text { que tu étais ma joie } \\
\text { et mon rêve brûlant } \\
\text { pour moi il n'y a plus de consolation } \\
\text { et c'est pourquoi je me saoule } \\
\text { pour oublier ton amour. }\end{array}$ \\
\hline
\end{tabular}




\begin{tabular}{|c|c|c|c|}
\hline 2 & B & $\begin{array}{l}\text { Quando voy a mi cotorro } \\
\text { lo veo desarreglado, } \\
\text { sólo, triste, abandonado, } \\
\text { me dan ganas de llorar } \\
\text { me detengo largo rato } \\
\text { campaneando tu retrato } \\
\text { pa' poderme consolar. }\end{array}$ & $\begin{array}{l}\text { Quand je rentre dans ma piaule } \\
\text { je la retrouve en désordre, } \\
\text { seule, triste, abandonnée, } \\
\text { ça me donne envie de pleurer } \\
\text { et je reste longtemps } \\
\text { à mater ton portrait } \\
\text { pour tenter de me consoler. }\end{array}$ \\
\hline 2bis & C & $\begin{array}{l}\text { Ya no hay en el bulín } \\
\text { aquellos lindos frasquitos } \\
\text { arreglados con moñitos } \\
\text { todos de un mismo color } \\
\text { y el espejo está empañado } \\
\text { y parece que ha llorado } \\
\text { por la ausencia de tu amor. }\end{array}$ & $\begin{array}{l}\text { Il n'y a plus dans la chambre } \\
\text { ces jolis petits flacons } \\
\text { décorés de rubans } \\
\text { tous de la même couleur } \\
\text { et le miroir est embué } \\
\text { on dirait qu'il a pleuré } \\
\text { l'absence de ton amour. }\end{array}$ \\
\hline 1 & D & $\begin{array}{l}\text { De noche cuando me acuesto } \\
\text { no puedo cerrar la puerta } \\
\text { porque dejándola abierta } \\
\text { me hago ilusión que volvés } \\
\text { siempre llevo bizcochitos } \\
\text { pa' tomar con matecitos } \\
\text { como si estuvieras vos } \\
\text { y si vieras la catrera } \\
\text { como se pone cabrera } \\
\text { cuando no nos ve a los dos. }\end{array}$ & $\begin{array}{l}\text { La nuit quand je me couche, } \\
\text { je ne peux pas fermer la porte, } \\
\text { car en la laissant ouverte } \\
\text { je me donne l'illusion que tu reviens } \\
\text { j'apporte toujours des biscuits } \\
\text { pour prendre avec le maté } \\
\text { comme si tu étais là } \\
\text { et si tu voyais le pieu } \\
\text { comme il est fâché } \\
\text { de ne plus nous voir tous les deux. }\end{array}$ \\
\hline 3 & $E$ & $\begin{array}{l}\text { La guitarra en el ropero } \\
\text { todavía está colgada, } \\
\text { nadie en ella canta nada } \\
\text { ni su cuerda hace vibrar } \\
\text { y la lámpara del cuarto } \\
\text { también tu ausencia ha sentido } \\
\text { porque su luz no ha querido } \\
\text { mi noche triste alumbrar. }\end{array}$ & $\begin{array}{l}\text { La guitare dans la penderie } \\
\text { est toujours à sa place } \\
\text { personne ne chante avec (en) elle } \\
\text { ni ne fait vibrer ses cordes } \\
\text { et la lampe de la chambre } \\
\text { elle aussi a senti ton absence, } \\
\text { car sa lumière n'a pas voulu } \\
\text { illuminer ma triste nuit. }\end{array}$ \\
\hline 1 & A & $\begin{array}{l}\text { Percanta que me amuraste } \\
\text { etc. }\end{array}$ & $\begin{array}{l}\text { Fille tu m'as abandonné } \\
\text { etc. }\end{array}$ \\
\hline
\end{tabular}

\section{Changements de rythme et tradition}

On reconnait des constantes musicales, rythmiques et poétiques de Villoldo à Gardel, bien que renouvelées et enrichies, ce qui nous permet de considérer qu'il y a innovation au sein d'une tradition chantée déjà établie par Villoldo.

Tout d'abord, on constate un certain ralentissement du tempo: El Pechador est joué andante (autour de 82 BPM) tandis que Mi Noche Triste est joué adagio (entre 74 et 
70 BPM). La musique de Castriota s'inscrit dans la tradition de la Guardia Vieja («Vieille Garde ») et est composée par le montage et l'arrangement de thèmes populaires de l'époque (Novati, 2008), comme celle de Villoldo. Mais le style diffère : ce morceau est exemplaire d'un moment dans l'esthétique du tango instrumental où non seulement le tempo ralentit, mais les mélodies s'écrivent de plus en plus en mode mineur. Cette transition est liée entre autres à la centralité grandissante du bandonéon qui remplace la flûte dans les orchestres (dits orquestas típicas), instrument de prestige qui, joué assis, ne permet pas autant de rapidité, et dont le souffle grave sonne comme une respiration haletante, un gémissement (Plisson, 2001). La musique instrumentale s'entend donc en elle-même comme une lamentation.

Il y a également continuité dans le motif rythmique assuré par la guitare, où nous retrouvons la cellule habanera. Mais le jeu de Ricardo enrichit le motif par des modulations du phrasé, des variations et des ornementations ${ }^{18}$, et par une grande maîtrise de la syncope, plus nettement marquée. Enfin la forme poétique présente elle aussi un allongement de la cadence. Si le vers octosyllabique traditionnel est conservé, aux couplets composés de doubles quatrains succèdent des strophes longues et disparates. On compte un dizain (la décima des chansons sentimentales latinoaméricaines) chanté sur la section 1 , suivi de deux septains chantés sur la section 2 , jouée deux fois, puis le retour du dizain avec la reprise de la section 1, et enfin un huitain sur la section 3 , avant la reprise du premier dizain sur la coda.

\section{Linéarité de la complainte}

18 Au-delà de ces changements de rythme localisés - versification, cellule rythmique de l'accompagnement, tempo - c'est le principe de répétitivité cyclique, constitutif du tango allègre, qui est abandonné. Le «rythme », au sens large de retour périodique du même, est bouleversé au niveau macrostructural. On identifie en effet toujours trois sections musicales, mais agencées selon un ordre inattendu et imprévisible (1-1-2-2bis-1-3-1). La section 1, répétée quatre fois après chaque changement de mélodie, sonne comme une épiphore mélodique. De plus, les mélodies 2 et 3 en sont des variations. Un jeu de répétition-variation est ainsi construit. La mélodie semble avancer par développement progressif (comme par reptations). Enfin, l'exposition est reprise en coda, ce qui crée un effet de fermeture. Ainsi, à une conception du rythme basée sur la régularité et la répétition, se substitue une construction linéaire, tendue vers une résolution finale.

19 Les couplets sont quant à eux à chaque fois différents, seul le premier est répété à la fin (A-B-C-D-E-A) : il s'agit d'une forme strophique (sans refrain), linéaire et non répétitive. Le poème semble être lui aussi sous-tendu par un principe de progression par la variation: le thème de l'abandon et du sentiment de vide qui lui est consubstantiel traverse le texte, un hyperthème ${ }^{19}$ annoncé dès le premier couplet et dont tous les couplets suivants déclinent un aspect. Une série d'objets personnifiés parcourt le poème, objets immobiles et permanents, comme figés dans l'attente de la femme aimée. Le poète semble tenter de remplir le vide par leur accumulation. L'hyperthème est évoqué aussi par les rimes, qui accentuent l'expression de la douleur par les rapports qu'elles établissent entre les mots de façon transversale, par exemple l'association entre vida et herida (A). D'autres rimes suggèrent plutôt des significations implicites, comme l'identité de forme entre alegría et quería (A), qui donne l'impression que la joie 
est conjuguée à l'imparfait, et imprègne le poème de nostalgie. De même vibrar rime avec alumbrar (E), établissant une correspondance entre la vibration - comme sonorité (par l'allitération en $[\mathrm{V}, \mathrm{B}, \mathrm{R}]$ ) et comme sensation - et la lumière, expérience positive qui est reléguée dans le passé et mise en opposition très nette avec l'inertie du présent sombre du locuteur. Dans toute récurrence s'insère donc une forme de décalage, permettant l'expression d'un malaise, d'un écart.

\section{Tensions-résolutions et dysphorie}

Autre fêlure du rythme, l'équilibre entre les parties est brisé. La section 2 (six mesures) est plus courte que la première et la troisième (huit mesures). Cela produit un effet d'accélération inscrit au cœur de la partition, et amène une tension qui est résolue dans la partie suivante par un retour à la normale en section 3. Ce jeu de tension/détente peut être considéré comme participant du rythme de la chanson au niveau macrostructural. Nous transposons ici l'analyse que Gustavo Beytelmann applique au jeu des musiciens dans le tango au niveau microstructural : selon lui la particularité du rythme tanguero est de déjouer en permanence la prévisibilité des récurrences par une action de tension/détente sur le schéma rythmique ${ }^{20}$. Ici, l'inégalité de durée entre les parties disloque l'uniformité de la chanson et crée un effet de surprise et de frottement, comme un resserrement de la respiration. On peut l'entendre comme le signifiant de l'angoisse manifestée dans les paroles chantées et le phrasé de Gardel : un «rythme de la mélancolie ", évoluant par répétitions-variations et par tensions-résolutions.

21 La tension monte jusqu'au moment d'acmé rythmique, mélodique et poétique qui conclut la section 3 : en effet l'oxymore mi noche triste alumbrar (E), oscillation entre le désespoir et l'espoir, conclut la seule section régulière dans le rapport vers/mesure, et un bref passage en mode majeur, immédiatement suivi d'un retour au mode mineur (via une cadence en mode mineur). Brève lueur d'espoir ou accentuation du désespoir ? Le retour en mineur a lieu justement sur le vers qui ouvre la possibilité d'une réconciliation, puisque l'infinitif présent (alumbrar), mode atemporel en espagnol comme en français, l'isole et le suspend hors du temps. C'est la reprise en coda qui semble résoudre cette suspension momentanée : ressassement, clôture sur le vers qui exhorte à l'oubli (olvidarme de tu amor) font définitivement basculer la chanson du côté du malaise.

\section{Dissociation des rythmes de la chanson}

Mais c'est aussi dans la dissociation entre les différents rythmes de la chanson que s'opère un basculement rythmique. Tout d'abord, la partie chantée et la partie instrumentale sont disjointes : l'accompagnement ne redouble plus la partie chantée, il accompagne au contraire le chanteur par un jeu de réponses et de contrepoints rythmiques, et harmonise la mélodie. Voix et guitare, complémentaires, s'entrelacent et dialoguent. Ensuite, l'homologie entre métrique et phrase musicale est brisée par la disjonction du vers et de la mesure. Le premier vers démarre avec une levée et se termine sur le premier temps musical à la reprise de la guitare : ainsi la pénultième syllabe du vers (amuraste), où se trouve l'accent tonique dans la langue parlée, tombe sur le premier temps de la mesure, le temps fort. Le cadre du vers et celui de la mesure sont par la suite constamment décalés de la sorte ${ }^{21}$. Nous retrouvons ici un autre jeu de tension/détente : mise en tension par le décalage, puis résolution, par exemple quand 
les quatre premiers vers de la section 1 , suivis d'un silence, sont finalement déployés sur l'équivalent de quatre mesures (seize temps). Dans la seconde partie de cette section (à partir de la cinquième mesure qui démarre sur quería), l'augmentation du nombre de vers par rapport au nombre de mesures contraint le chanteur à une accélération du débit : six vers sont enchaînés sur seize temps, séparés par une seule pause. Autre tension, autre résolution : la fin du couplet coïncide in fine avec la fin de la période musicale ${ }^{22}$. Le même phénomène se produit dans la section 2 , mais accru, avec sept vers à chanter sur six mesures. La tension entre le chant et l'instrument augmente, suivie d'une résolution d'autant plus spectaculaire à l'entrée de la section 3, qui, elle, amène un temps calme avec le retour à une cadence régulière. Retour à l'équilibre provisoire, elle fait le pont avant la coda, la tempête finale.

Dernier décalage, celui du phrasé de Gardel, qui joue en permanence avec le cadre du vers et avec la cellule rythmique de base, qu'il anticipe ou déborde: contretemps, syncopes inattendues créent des déséquilibres, mais très contrôlés, ce qui lui permet de retomber sur ses pieds à chaque fin de période. Par ces jeux de tension/détente, il dirige à sa guise le déroulement de la chanson dans un flux irrégulier. Cette interprétation opère un point de bascule d'une conception du rythme à une autre. Gardel s'approprie la partition pour proposer une interprétation singulière et s'inscrit dans un 'faire' inimitable. La chanson devient performance au sens spectaculaire du terme. Mais surtout, son phrasé rubato apporte une dimension d'imprévisibilité à cette chanson, qui met en déroute l'horizon d'attente de l'auditeur. Dès son entrée en voix, Gardel prolonge la levée, longue pause qui coïncide avec la césure du vers (sur que) et la note la plus haute. La guitare attend. Ce débit affirme l'autorité d'une voix dynamique et montante, en parfaite maîtrise de la situation, et cela sur un vers qui peut s'entendre au départ comme une prise de parole querelleuse: le percanta, terme familier en lunfardo ("gonzesse ", " cocotte»), s'entend, associé au que... menaçant, comme une insulte ("salope »). La suspension du débit qui suit rend la chute du vers d'autant plus surprenante : le caïd est retourné en victime de l'amour (le que... devient plaintif). Les valeurs et relations de genre propres au tango " canaille » sont inversées. À la brisure $d u$ rythme correspond donc un éclatement du sens. Ces changements de rythme installés au sein de la chanson opèrent en contrepoint de l'attente figée que véhicule le texte inerte. La dynamique de la voix agit, agite la parole, donne sens - c'est-à-dire mouvement - aux paroles. Comme l'écrivait Paul Zumthor (1982, 235), «la voix [...] constitue (plus qu'elle ne transmet) un message ».

\section{Chanter la mélancolie}

C'est aussi par son style vocal que Gardel marque une rupture importante par rapport à Villoldo: voix de fausset, grande maîtrise du vibrato, trémolos, ornementations, mélismes et variations d'intensité, sont autant de moyens expressifs qu'il emprunte (en autodidacte) à des styles savants comme le bel canto et l'opéra. Sa diction reste syllabique, mais loin du syllabisme «à la lettre » de Villoldo. De même que le son du bandonéon devient le son de la lamentation tanguera, la vocalité vibrante de Gardel devient la voix du tango, la seule façon de dire la souffrance tanguera. Cette dramatisation de la voix au service de la complainte est combinée, dans les images et vidéos que nous avons de Gardel, à une expression contractée du visage, les yeux fermés, le front plissé, manifestant la douleur. D'un point de vue pragmatique, la performance dans son ensemble induit une émotion mélancolique, qui convient bien à 
la position d'écoute assise du public immobile. Gardel institue sa façon de chanter, qui devient propre au tango. Au point que certains tangueros considèrent que l'on ne doit pas danser sur une chanson mélancolique : c'est une « pensée triste »... qui s'écoute ${ }^{23}$.

La dysphorie, présente dans la forme même du chant par les nombreux bousculements rythmiques, et véhiculée par la voix sanglotante, n'est finalement, dans l'expérience de la performance, que redite par le sens des mots. Le texte chanté présente bien des motifs de la mélancolie poétique. On retrouve des topoï de la poésie moderniste (Balderston, 1989), comme l'identification à la grande ville moderne. Villoldo inscrivait déjà sa chanson dans l'espace de la ville, mais un déplacement est effectué ici : le texte est rattaché à l'univers de la mauvaise vie de Buenos Aires par les termes de lunfardo (percanta, cotorro, bulín, campanear), mais à un niveau minimal, sans représenter une scène faubourienne. Ce cadre aux contours brouillés, incertains, est prétexte à l'évocation de la souffrance amoureuse. Alors que Villoldo cherchait à incarner le faubourg, Contursi s'en échappe et ne l'évoque que pour s'en abstraire. De plus, la chanson présente un "je » lyrique et introspectif, isolé dans sa chambre et dans sa singularité. Mais c'est aussi un "je » désincarné, aux contours flous : il n'est jamais nommé, contraste frappant avec notre Pechador aux trois noms. Enfin, la chanson de Villoldo s'appuyait entièrement sur le présent et le mode assertif, signes d'une réalité certaine (yo lo puedo asegurar), célébration du présent. Ici au contraire le «je »-chanté est tourné vers le passé, entre nostalgie d'un amour radieux et rejet d'un présent solitaire. Au-delà de toute opposition rigide entre vérité et fiction, Gardel chante ce «je » en emplissant sa voix d'une émotion telle que c'est comme si c'était lui. En somme, la relation au public ne s'établit plus autour du texte, mais autour de la musique. C'est la voix chantante de Gardel qui suscite l'émotion, au point que la chanson peut émouvoir jusqu'aux pleurs celui qui ne comprend pas les paroles ${ }^{24}$.

\section{Conclusion}

Le rythme cyclique de Villoldo a été fêlé par la grande liberté vocale de Gardel, qui joue avec le vers. Maints traits structurants sont conservés, mais la disjonction entre poème et musique et le phrasé accidenté de Gardel sont autant de secousses qui permettent de dire la mélancolie. On pourrait le formuler ainsi : la chanson de Gardel se situe dans la continuité de celle de Villoldo pour tout ce qui touche à la régularité, mais elle rompt avec elle en insérant l'irrégulier dans cette régularité. C'est le paradoxe de ce "rythme de la mélancolie » : l'entrain et le dialogisme de la chanson de Villoldo sont conformés dans un phrasé au rythme régulier ; tandis que le soliloque mélancolique de Gardel est projeté dans un phrasé changeant, fondé sur l'écart et l'imprévisibilité. Cette imprévisibilité n'est possible que parce que la configuration de la performance est différente. Mettre en déroute - sentimentale ou rythmique - un danseur est un interdit du tango ; mais un auditeur assis, cela devient possible. La transformation rythmique, du rapide au lent et du régulier à l'irrégulier, le changement de registre, de l'entrain à l'amertume, et le déplacement topologique et poétique des marges au centre, sont indissociables. Sans cette approche globale de la chanson comme acte multiforme, on manquerait ce qui constitue justement ces rythmes chantés - ce qu'Henri Meschonnic a appelé "mouvement », " changement ", «flux » et "sens du sujet dans le discours " (2009), impliquant de penser le passage du texte inerte à l'interprétation vocale qui lui donne mouvement, corps et sens. Bel exemple que le phrasé de Gardel, contradiction 
du poème écrit, déchaînant la tonalité monotone par cette temporalité imprévisible. Meschonnic opposait le rythme, espace de la subjectivité et de l'individuel, à la mesure, expression de la norme (Ibid.). Dans cette optique, l'interprétation de Gardel est la mise en branle par le sujet chantant de la partition normative. La mélancolie chantée éloigne le tango d'une concordance parfaite entre les différents rythmes de la chanson, euphonie propre à chanter l'euphorie, pour aller vers l'expression d'une discordance, d'un heurt ${ }^{25}$.

\section{BIBLIOGRAPHIE}

BALDERSTON, Daniel, 1989, Celedonio Flores's ‘Sonatina’: Lunfardo Parody and Post-Modernist Esthetics, Hispania, vol. 72, pp. 123-129.

BINDA, Enrique, (s.d.), Bandas, rondallas y tangos, www.todotango.com [consulté le 21 avril 2015].

BINDA, Enrique, (s.d.), ¿A que velocidad se grabaron los 78?, www.todotango.com [consulté le 21 avril 2015].

BINDA, Enrique y García BRUNELLI, Omar, 2012, El problema de la velocidad de los discos de $78 \mathrm{rpm}$. Su incidencia en la historia estética del tango, 9 août 2012, Programa de Salvaguardia de Tango/ Publicaciones, Centro Feca [consulté le 21 avril 2015].

BRIAND, René, 1972, Crónicas del tango alegre, Buenos Aires, Centro Editor de América Latina.

CALAME, Claude, DUPONT, Florence, LORTAT-JACOB, Bernard, MANCA, Maria (dir.), 2010, la Voix actée : pour une nouvelle ethnopoétique, Paris, Kimé.

CEPITELLI, Mathieu, 2011, la Question du rythme dans le répertoire pour sexteto típico des années 1920-1930, propositions pour une analyse du rythme dans la musique tango. Colloque international « Tango : Création/Identification/Circulation », Paris, EHESS, octobre 2011. CHABOT-CANET, Céline, 2008, Léo Ferré: une voix et un phrasé emblématiques, Paris, L'Harmattan. GOBELLO, José, 2003, Nuevo diccionario lunfardo, Buenos Aires, Corregidor. GOBELLO, José, 2004, Letras de tango, Selección (1897-1981), Buenos Aires, Meralma. MESCHONNIC, Henri, 2009, Critique du rythme : anthropologie historique du langage, Lagrasse, Verdier

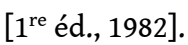

NOVATI, Jorge (dir.), 2008, Antología del tango rioplatense, vol. 1 : Desde sus comienzos hasta 1920, Buenos Aires, Instituto Nacional de Musicología Carlos Vega [1 $1^{\mathrm{a}}$ ed., 1980].

PELINSKI, Ramón, 1981, Dire le tango, Études françaises, vol. 17, p. 111-136.

PELINSKI, Ramón, 2000, Invitación a la etnomusicología. Quince fragmentos y un tango, Madrid, Akal Ediciones.

PLISSON, Michel, 2001, Tango, du noir au blanc, Paris, Cité de la musique/Arles, Actes Sud.

PUCCIA, Enrique Horacio, 1976, El Buenos Aires de Angel G. Villoldo, 1860-1919, Buenos Aires, Películas Impulso. 
REY DE GUIDO, Carla y GUIDO, Walter, 1989, Cancionero rioplatense, 1880-1925, Buenos Aires, Biblioteca Ayacucho.

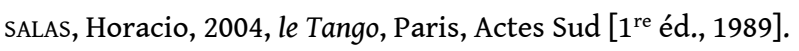

ZUMTHOR, Paul, 1982, Considérations sur les valeurs de la voix, Cahiers de civilisation médiévale, vol. 25, p. 233-238.

\section{NOTES}

1. Sur la définition du tango « savant » (appelé tango letrado en espagnol), voir PELINSKI (2000).

2. Voir SALAS (2004) et GOBELlo (2004). Ce dernier considère les "petites paroles" (letrillas) de Villoldo comme du «tango préhistorique » et fait démarrer la tradition du tango-chanson avec Contursi en 1917.

3. Terme emprunté à Zineb MAJDOULI (intervention au séminaire du GREP, CERILAC, ParisDiderot, 09/10/2012).

4. «Portègne » (porteño), qui vient du port de Buenos Aires et, par extension, de Buenos Aires.

5. Qualificatif qui apparaît dans plusieurs chansons, et déjà repris par BRIAND (1972) et PUCCIA (1976).

6. Villoldo a enregistré cette chanson à trois reprises: en 1907 chez Odeón ; en 1909-1910 chez Era (matrice 60279 ) con banda de música; et en 1909-1910 chez Columbia avec l'Orquesta Heyberger (d'après Enrique Binda, échange inédit). Voir BINDA, Bandas, rondallas y tangos (s.d.).

7. L'analyse doit prendre en compte les défauts de l'enregistrement, qui ont altéré certaines caractéristiques instrumentales et vocales de l'interprétation, comme la voix de Villoldo qui paraît plus fausse et nasillarde qu'elle ne l'est en réalité. Voir BINDA, ¿A que velocidad se grabaron los 78 ? (s.d.) ainsi que BINDA et BRUNELLI (à paraître).

8. A contrario, les interludes instrumentaux sont, eux, harmoniques.

9. Ou de type polka, la cellule habanera s'inscrivant alors comme variante. On entend l'interpénétration de plusieurs rythmes binaires, exemplaire des synthèses musicales de l'époque. Voir PLISSON (2001) et NOVATI (2008).

10. Néologisme qui élargit le terme « métapoétique » au chant (poésie + vocalisation).

11. Le lexique lunfardo est traduit d'après Gobello (2003), complété du dictionnaire en ligne sur le site todotango.com. Nous avons traduit chulinga par « flûte » et chimango par « écervelé » afin de conserver l'isotopie de l'oiseau.

12. La zarzuela est un théâtre populaire très en vogue à l'époque dans le río de la Plata. On y chante souvent des «tangos ». Villoldo lui-même a écrit des chansons pour ce genre théâtral (Rey de Guido y Guido, 1989).

13. C'est ainsi que nous proposons de l'appeler dans notre thèse de doctorat (en cours, université Paris-Diderot).

14. Le «style scandé » est opposé à la «prosodie libre » par JAKOBSON (1963), cité par CHABOT-CANET $(2008,16)$. 
15. Le jeu ne se réduisait sans doute pas à la seule mise en voix : on peut imaginer toute une gestuelle comique et une expressivité du visage associées à la chanson allègre, comme celles des chanteuses célèbres qui reprendront ses chansons. Mais, en l'absence d'archives, ce ne sont là que conjectures.

16. Disque Max GLUCKSMAN ( ${ }^{\circ} 18010$ matrice 89), 1917). Il l'enregistrera de nouveau en 1930 (Ibid.).

17. "Incorporée ", traduction limitée du mot anglais embodied : c'est-à-dire à la fois reconnue par la collectivité des participants du fait de l'adresse directe au public, et corporellement appropriée par le rire, par la mémorisation, la reprise du refrain, et même le balancement, voire la danse.

18. Par exemple : croche pointée + double croche suivie d'une quadruple croche.

19. L'hyperthème en stylistique de la phrase est le thème qui traverse et organise le texte entier. On parle de rapport thème-propos (chaque couplet est un propos sur le thème principal) et de progression à thème constant.

20. D'après CEPITELLI (2011).

21. Si on transcrit la partition à partir de la performance enregistrée, on est invité à l'écrire en $4 / 4$, comme pour Villoldo, et à compter les temps à partir de la reprise de la guitare après la pause imposée par l'entrée du chant.

22. On notera que durant cette accélération, dans la seconde partie de la section 2 , l'accompagnement se dédouble en deux parties distinctes (deux guitares), comme dans l'introduction, décuplant la tension entre les voix.

23. Déformation du célèbre mot du compositeur Enrique Santos Discépolo pour qui le tango est « une pensée triste qui se danse ».

24. Nous en avons fait l'expérience en faisant écouter cette chanson à plusieurs auditeurs non hispanophones, qui reconnaissent immédiatement la chanson comme complainte et comme donnant en partage une émotion triste.

25. Nous remercions infiniment Bernard Lortat-Jacob, Gregory Beller, Matias Isolabella, Omar García Brunelli et Enrique Binda pour leur aide précieuse, ainsi que Maria Manca et Sandra Bornand (du GREP) pour leurs relectures.

\section{RÉSUMÉS}

Quand on compare les chansons tango El Pechadord'Ángel Villoldo (1909) et Mi Noche Triste de Carlos Gardel (1917), on entend le passage d'un style allègre à un style mélancolique. Cette transformation historique, où le rythme est central, s'articule à un changement dans la configuration des performances. Le tango allègre de Villoldo est caractérisé par une forme cyclique reposant sur la répétition et la redondance, facilement mémorisable et permettant donc une participation active de l'auditeur dans le cadre de fêtes populaires. Villoldo se met en scène dans un texte comique visant à provoquer le rire. Son interprétation vocale, forme de parléchanté, est régulière et mimétique. La chanson de Gardel s'inscrit par plusieurs aspects dans la continuité de celle de Villoldo, notamment par certaines constantes rythmiques. Mais au style 
euphonique du premier s'oppose le style dysphorique du second : Gardel offre le spectacle de son chant mélancolique à une assemblée assise. La chanson devient alors lyrique, comme cela a souvent été vu, mais aussi linéaire, reposant sur un principe de variation et contrariant les attentes de l'auditeur par des jeux rythmiques de tension-résolution. Son phrasé, quant à lui, devient imprévisible. L'écart entre ces deux performances chantées permet de souligner les corrélations entre un style, un type de rythme, un registre poétique et une situation pragmatique.

Comparison between the tango songs El Pechador by Angel Villoldo (1909) and Mi Noche Triste by Carlos Gardel (1917) stresses the change from a light-hearted style to a melancholic style. This historical transformation, in which rhythm is central, is related to a change in the layout of performances. Villoldo's lively tango is defined by a cyclical form based on repetition and redundancy and easy to remember, thus encouraging active participation of the audience in contexts of popular festivities. Villoldo sketches himself in a comical text designed to provoke laughter. His vocal performance, a spoken-sung form, is regular and mimetic. Gardel's song is in many ways a continuation of Villoldo's, reproducing in particular some rhythmical patterns. But the euphonious style of the first is opposed to the dysphonic style of the second: Gardel offers the spectacle of his melancholic song to a seated assembly. The song then becomes lyrical, as it has often been observed, but also linear, based on variation and thwarting the listener's expectations through rhythmic plays of tension-resolution. As to his phrasing, it becomes unpredictable. The gap between these two kinds of sung performances emphasizes the correlation between style, rhythm, poetics and pragmatic context.

\section{INDEX}

Keywords : Song, Tango, Melancholy, Speech, Voice, Ethnopoetic

Thèmes : ethnomusicologie, ethnopoétique

Index géographique : Argentine

Mots-clés : chanson, tango, mélancolie, paroles, voix

\section{AUTEUR}

\section{PÉNÉLOPE PATRIX}

CERILAC, Université Paris-Diderot 